\title{
Diseño e Implementación de un Sistema de Regulación de Carga de Batería en una Planta de Microgeneración Hidráulica
}

\author{
Design and Implementation of a Battery Charge Regulation System in a Hydraulic Microgeneration Plant
} Emil Hernández Arroyo ${ }^{1 *}$, Omar Pinzón Ardila ${ }^{1}$ y Alfonso Santos Jaimes ${ }^{1}$

\begin{abstract}
Resumen
En este proyecto se diseña y construye un sistema de regulación de carga de batería en una planta de microgeneración hidráulica que usa una turbina de reacción tipo Francis. Para regular la carga de la batería se adapta un controlador MPPT [Maximum Power Point Tracker] el cual se utiliza en sistemas fotovoltaicos. La turbina convierte la energía hidráulica en energía mecánica y el generador transforma la energía mecánica en energía eléctrica, la cual se rectifica a 12 V DC (Direct Current) para alimentar el controlador que regula la carga de la batería. La energía hidráulica se genera con una bomba centrifuga de $5.5 \mathrm{Hp}$ y proporciona una altura o cabeza equivalente a $28 \mathrm{~m}$. Se utiliza una batería de $12 \mathrm{~V} / 100$ Ah y es cargada por el sistema en $24 \mathrm{~h}$, la eficiencia del generador es aproximadamente $75 \%$.

Abstract

In this project, a battery charge regulation system was designed and built using a microgeneration plant with a Francis type turbine. To regulate the charge of the battery an MPPT (Maximum Power Point Tracker) controller was adapted which is used in photovoltaic systems. The turbine converts the hydraulic energy into mechanical energy and the generator transforms the mechanical energy into electrical energy, which was rectified at $12 \mathrm{~V}$ DC [Direct Current] and the controller regulates the charge of the battery. The hydraulic power was generated with a centrifugal pump of $5.5 \mathrm{Hp}$ and it provides a height or head equivalent to $28 \mathrm{~m}$. In this setup a $12 \mathrm{~V} / 100 \mathrm{Ah}$ battery was charged by the system in $24 \mathrm{~h}$ and the generator efficiency is approximately $75 \%$.
\end{abstract}

\section{Palabras Clave}

Turbina hidráulica; controlador de carga; bomba centrifuga; batería.

Key words

Hydraulic turbine; load controller; centrifugal pump; battery.

${ }^{1}$ Facultad de ingenierías, Universidad Pontificia Bolivariana, Colombia

*Autor correspondiente: emil.hernandez@upb.edu.co

Manuscrito recibido 24-03-2017; revisado 11-04-2017; aceptado 26-04-2017.

\section{Introducción}

Las turbinas son comúnmente usadas en sistemas de microhidropotencia. El agua en movimiento impacta los álabes de la turbina, en la mayoría de los casos como en una rueda hidráulica produciendo el giro de un eje. Sin embargo, las turbinas son más compactas conforme aumenta la energía de salida de las ruedas hidráulicas. Estás ruedas, tienen pocos engranajes y requieren menos material para su construcción [1]. Las baterías son el tanque de almacenamiento de potencia de los sistemas solares e hídricos, además suministran potencia cuando hay ausencia del sol [2].

Es por ello, que existen numerosas investigaciones sobre sis- temas de control de carga en baterías, como, por ejemplo, Yasser y Naggar [2], quienes diseñan e implementan un controlador utilizando una técnica MPPT, un algoritmo genético y una estrategia de corriente y voltaje constante. Estas modificaciones reducen apreciablemente el periodo de carga de la batería. Los resultados experimentales muestran que el nuevo controlador tiene una mejora significativa y una mayor eficiencia comparado con el controlador tradicional.

Las turbinas microhidro cargan las baterías continuamente, es por ello que se requiere que en todos los sistemas las baterías se carguen completamente en algún momento. Por lo tanto, se necesita detener la carga para prevenir la sobrecarga, de hecho, en los sistemas microhidro esta situación se puede presentar 
con frecuencia [3].

Shengjun [4] presenta un diseño de una estrategia de control para cargar vehículos eléctricos basados en generación fotovoltaica, baterías de almacenamiento y conexión a red. La estrategia de optimización es útil para resolver la integración de sistemas de potencia distribuidos a gran escala y los problemas de carga de vehículos eléctricos en conexión a red.

Sadid y colaboradores [5] propone una formulación matemática para modelar el costo de incertidumbre en la operación de una pequeña central hidroeléctrica, partiendo de las formulaciones propuestas para sistemas solares y eólicos.

\section{Teoría}

\subsection{Calculo de potencia}

La potencia disponible o potencia hidráulica en un sistema de microgeneración hidráulica se calcula con la (1) [6].

$$
P h=\gamma Q H n
$$

$\gamma:$ peso específico del agua $\left[N / \mathrm{m}^{3}\right]$

$Q:$ Caudal $\left[\mathrm{m}^{3} / \mathrm{s}\right]$

$H n$ : Altura neta $[m]$

$P h$ : Potencia hidráulica $[W]$

La altura neta se calcula con la Ecuación (2).

$$
H n=P n / \gamma
$$

$P n$ : presión neta $[\mathrm{Pa}]$ : Presión cuando hay flujo de agua. La potencia de accionamiento o potencia de eje se calcula con la (3).

$$
P e j e=P h * \eta t
$$

$\eta t$ : eficiencia de la turbina

La potencia de salida o potencia del generador se calcula con la Ecuación (4).

$$
\text { Pgen. }=\text { Peje } * \eta \text { gen } .
$$

$\eta$ gen: eficiencia del generador

En la Figura 1, con una $P n=206000 P a, \gamma=9880 \mathrm{~N} / \mathrm{m}^{3}$, $\eta t=0.75 y \eta$ gen $=0.6$ se obtiene:

$P h=1659.8 W$ Peje $=1244.8 W$ Pgen.$=746.9 \mathrm{~W}$

\subsection{Dimensionamiento de la tubería forzada}

La tubería forzada es el tramo de tubería que se extiende desde la fuente hídrica hasta la turbina [7].

La altura geométrica se calcula con la (5).
Figura 1. Sistema de microgeneración hidráulica.

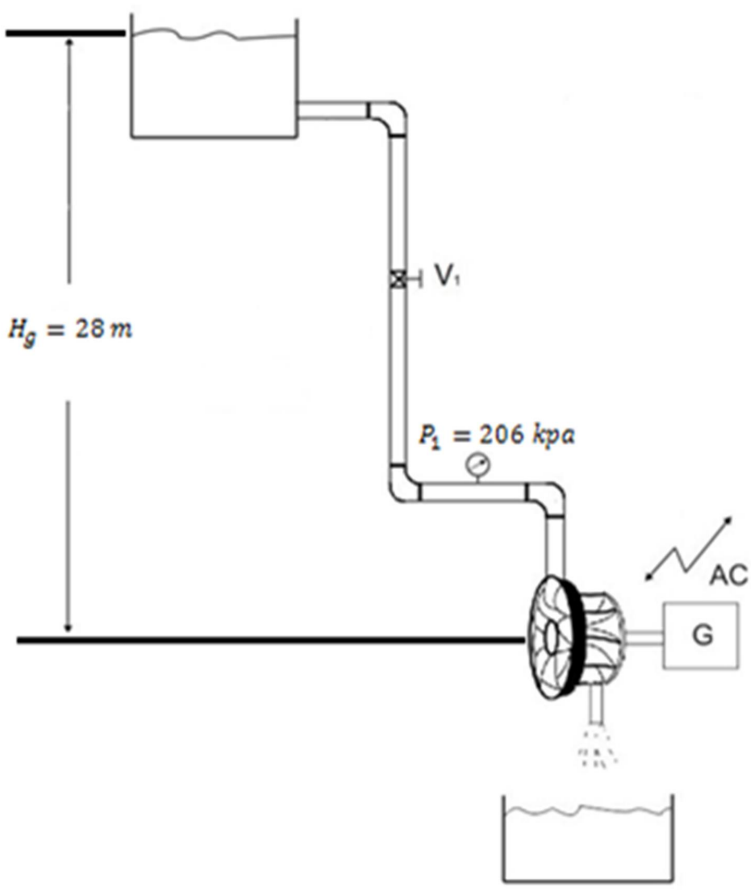

Tabla 1

Características de la tubería forzada.

\begin{tabular}{lc}
\hline \hline Diámetro interno & 3 pulgadas \\
Caudal óptimo & $225 \mathrm{gpm}$ \\
Potencia hidráulica & $3 \mathrm{~kW}$ \\
Tipo de tubería & $p v c$ \\
\hline \hline
\end{tabular}

La pérdida vertical ideal se calcula con la Ecuación (6) y (3).

$$
\text { Perdidaverticalideal }=0.25 * H g
$$

Para un $H n=21 m$ y usando la Ecuación (5) y (6) se obtiene una perdida vertical ideal de $7 m$.

La pérdida de cabeza por fricción se obtiene de la Ecuación (7) [8].
Perdidavertical $=\mathrm{Hg}-\mathrm{Hn}$ 
Figura 2. Estructura del sistema hidráulico.

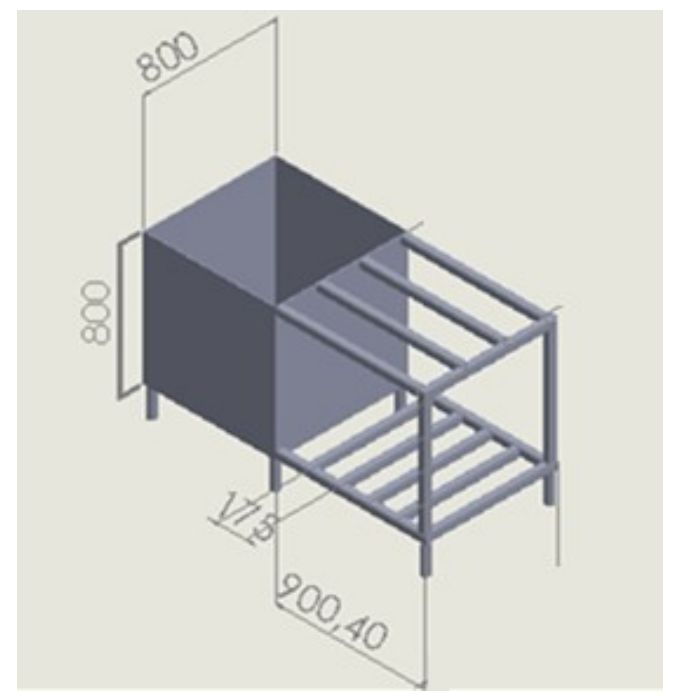

\section{Metodología}

\subsection{Estructura geométrica}

Teniendo en cuenta las dimensiones de los elementos que componen el banco de micro generación hidráulica se diseña una estructura que soporte la carga de los elementos que la componen y que permita su fácil manejo al momento de poner en marcha. Se utiliza acero cold roll en láminas y tubos de sección cuadrada de diferentes calibres. La estructura cuenta con un tanque de almacenamiento de agua con láminas de calibre 0.25 pulgadas, tubería cuadrada de $2 \times 2$ "(pulgadas) tipo pesado de cold roll 1020 y 6 ruedas giratorias con freno de $200 \mathrm{~kg}$. En la Figura 2 se muestra el plano geométrico del banco utilizando el software Solidworks, las dimensiones están en mm.

\subsection{Diseño hidráulico}

En la Figura 3, se muestra el esquema para la construcción del sistema hidráulico donde se utiliza una bomba centrífuga que simula la cabeza [9, 10], una turbina de reacción tipo Francis, con una potencia disponible o potencia hidráulica de $1.4 \mathrm{~kW}$. Se asume una eficiencia de la turbina de $60 \%$ para obtener una potencia en el eje o potencia de salida de $1.06 \mathrm{~kW}$ y una eficiencia del generador de $75 \%$ para obtener una potencia eléctrica de $0.64 \mathrm{~kW}$.

Los valores de potencia de la Figura 3, corresponden a un $H n=18 m$ con una presión neta de $176 \mathrm{kPa}$.

Las Figuras 4 y 5 presentan fotografías del banco de microgeneración donde se observan la distribución de los principales elementos que lo componen.

\subsection{Diseño eléctrico}

El controlador MPPT permite regular una tensión variable en la entrada para entregar en la salida una tensión acondiciona para cargar el sistema de baterías.
Figura 3. Esquema del sistema de microgeneración hidráulica.

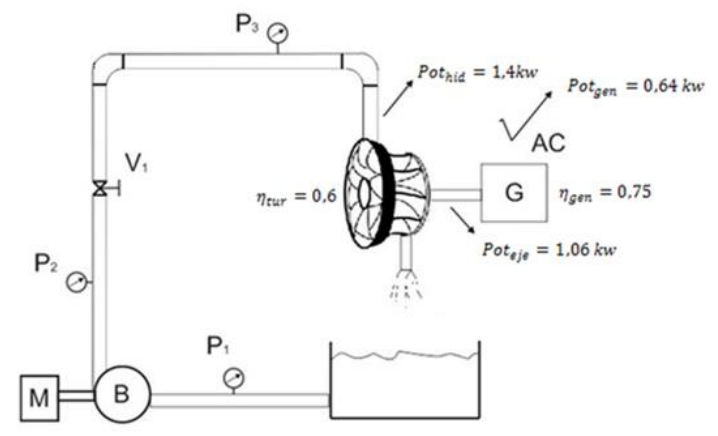

Figura 4. Banco de microgeneración hidráulica construido.

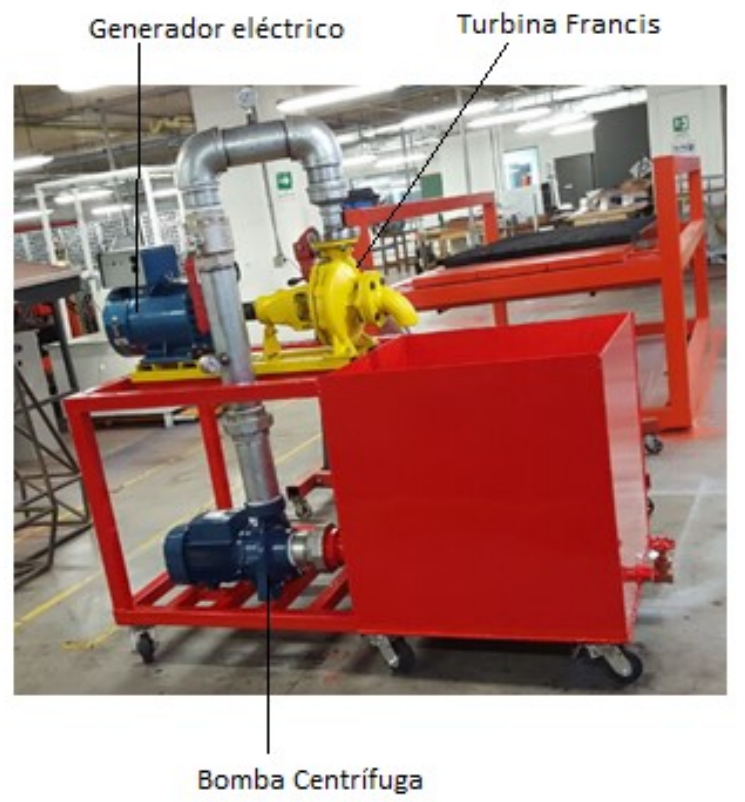

En la Figura 6 se muestra el diagrama eléctrico donde el generador entrega una tensión variable (220VAC, nominales), la cual se reduce a $24 V A C$. Un puente de diodos se utilizan para conseguir $12 V D C$ y por último la señal se filtra con dos condensadores de $1000 \mu F$ y $100 V$ previa conexión al controlador MPPT75/15 que optimiza la carga de las baterías y desvía la energía a una carga resistiva de $240 \mathrm{~W}$ cuando la batería se cargan en su totalidad.

Los siguientes datos corresponden a las especificaciones de placa de los elementos que componen el sistema eléctrico según las Tablas 1, 2, 3 y 4.

Un controlador de carga es una parte esencial de cualquier sistema que carga baterías, por fuentes fotovoltaicas, eólicas, hidráulicas, fuel o redes públicas. Su propósito es mantener las baterías alimentadas de forma segura y a largo plazo. Los controladores bloquean la corriente inversa y previenen la sobrecarga de las baterías. Algunos controladores previenen la sobre descarga, protegen de la sobrecarga eléctrica, además 
Figura 5. Banco de microgeneración hidráulica con el sistema eléctrico.

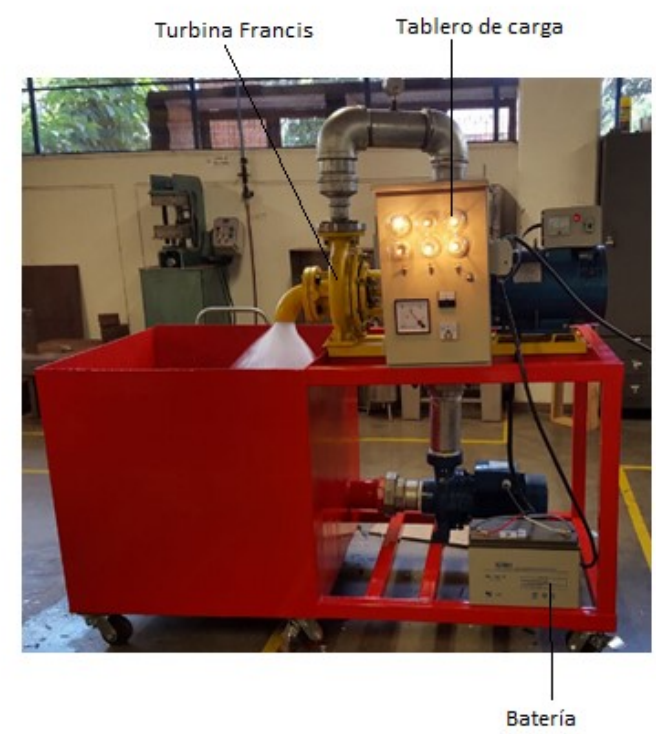

Tabla 2

Especificaciones del controlador.

\begin{tabular}{lc}
\hline \hline \multicolumn{2}{c}{ Controlador MPPT $75 / 15$} \\
\hline Tensión de la batería [V] & $12 \mathrm{~V} / 24 \mathrm{~V}$ \\
Corriente máxima batería [A] & $15 \mathrm{~A}$ \\
Eficiencia máxima & $98 \%$ \\
Autoconsumo [mA] & $10 \mathrm{~mA}$ \\
Potencia máxima $P V / 12 \mathrm{~V}$ & $200 \mathrm{~W}$ \\
\hline \hline
\end{tabular}

muestran el estado de la batería.

El puente, junto con un condensador y un diodo zener, limitan la tensión, permitiendo la conversión segura de la corriente alterna a la corriente continua. La función de los cuatro diodos es lograr que la corriente circule en un solo sentido, mientras que el resto de componentes tienen como función estabilizar la señal.

\section{Resultados}

En la Figura 7 se observa el funcionamiento de la turbina respecto al caudal y las revoluciones. El caudal se modifica utilizando una válvula tipo globo con un ángulo apertura de $90^{\circ}$ y las cinco posiciones parten del shut off o posición $0^{\circ} \mathrm{y}$ las siguiente cuatro aperturas se consiguen cada $22,5^{\circ}$ siendo la última posición full open o posición $90^{\circ}$.

En la Figura 8 se observa que en las dos primeras aperturas de la válvula la potencia de salida o generada es muy baja mientras que las revoluciones aumenta significativamente. En las siguientes tres muestras la potencia generada aumenta consid-
Figura 6. Diagrama eléctrico del sistema de carga de batería.

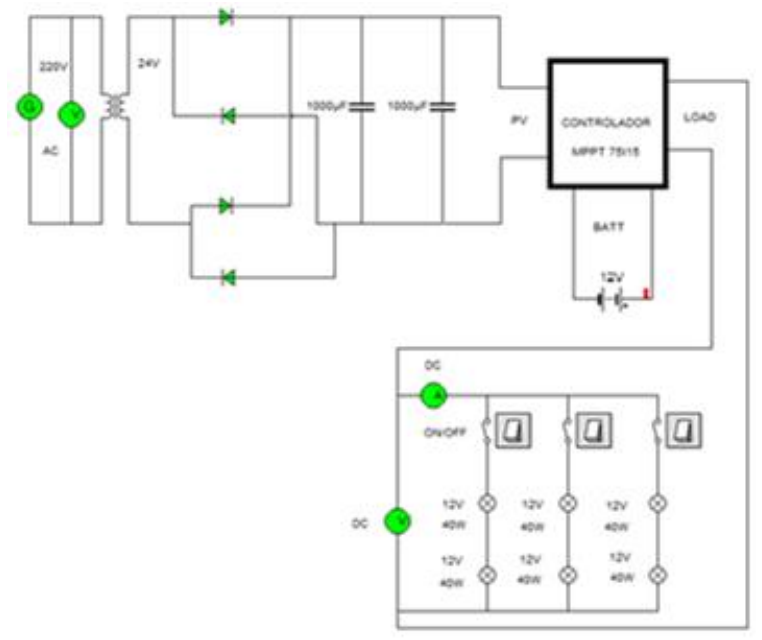

Tabla 3

Especificaciones del puente de diodos.

\begin{tabular}{lc}
\hline \hline \multicolumn{2}{c}{ Puente de diodos KBPC5010 } \\
\hline Corriente en directo máxima [A] & 50 \\
Voltaje en directo por elemento [V] & 1.2 \\
Voltaje DC inverso máximo [V] & 1000 \\
Corriente pico en directo [A] & 400 \\
\hline \hline
\end{tabular}

erablemente, lo que permite concluir que, a partir de 980rpm, el generador eleva la potencia generada, consiguiendo que el funcionamiento ideal del generador se encuentra entre $1600 \mathrm{y}$ 1800 rpm.

La Figura 9 muestra una carga inicial del acumulador de 11,3 Voltios, transcurridos 24 horas, el voltaje de la batería es 13.7 voltios, tiempo de carga de la batería.

En esta prueba se evidencian las tres etapas de carga de una batería, la primera etapa es la etapa inicial (bulk), durante esta fase, el controlador suministra tanta corriente de carga cómo es posible para cargar la batería rápidamente, en esta fase el controlador suministra corriente entre los $6 A$ y $7 A$ para cargar la batería hasta un voltaje de 14.2 voltios. Cuando la tensión de la batería alcanza la tensión de absorción predeterminada, el controlador activa la siguiente fase (fase de absorción), en ésta el controlador conmuta a un modo de tensión constante, aplicando a la batería una corriente de carga entre los $2 A$ y

\section{Tabla 4}

Especificaciones de la batería.

\begin{tabular}{lc}
\hline \multicolumn{2}{c}{ Batería } \\
\hline Voltaje nominal [V] & 12 \\
Voltaje en directo por elemento [V] & 1.2 \\
Voltaje DC inverso máximo [V] & 1000 \\
Corriente pico en directo [A] & 400 \\
\hline \hline
\end{tabular}


Figura 7. Caudal vs Rpm en la turbina Francis.

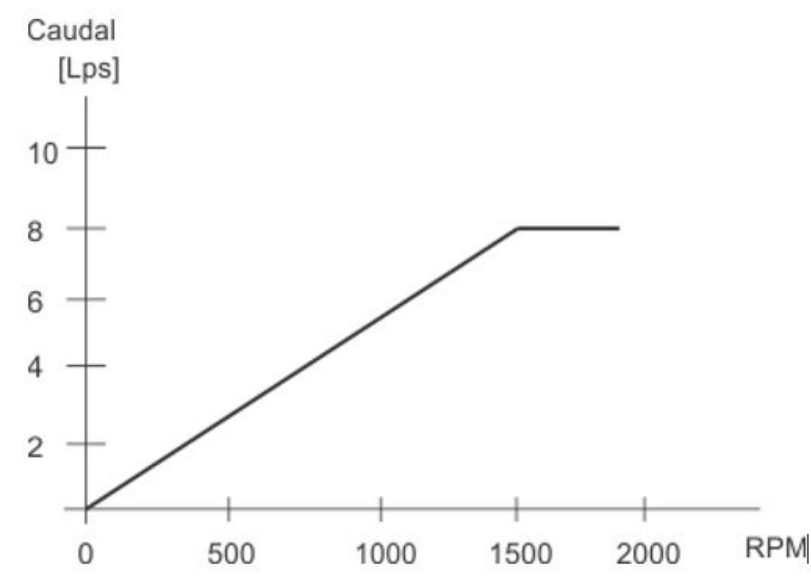

Figura 8. Potencia generada vs Rpm.

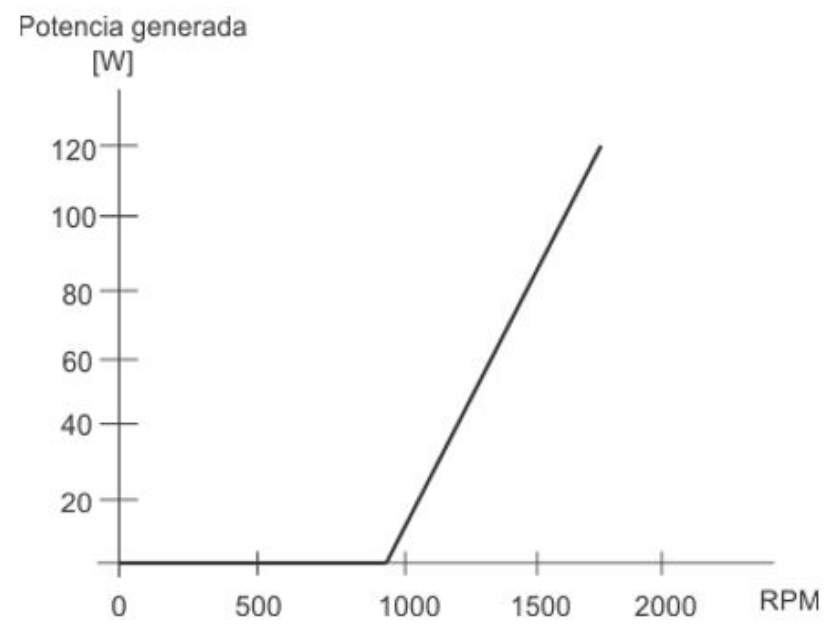

4A. Cuando la corriente de carga disminuye hasta alcanzar la corriente predeterminada de transición a carga de flotación, la batería está completamente cargada y el controlador cambia a la siguiente fase (fase de flotación), durante esta fase se aplica la tensión de flotación, 13.7 voltios a la batería para mantenerla completamente cargada. Cuando la tensión de la batería desciende por debajo de 13,2 voltios durante al menos 1 minuto, se inicia un nuevo ciclo de carga.

En la Figura 10 se observa el comportamiento de la batería con una potencia de carga constante sin recibir energía. La batería del estudio es de $12 \mathrm{~V}$ - 100 Amperios hora [Ah], se selecciona una carga resistiva de $240 \mathrm{~W}$, ubicadas en el tablero de control. Además, en la Figura 10 se muestra una carga inicial del acumulador de $13,7 \mathrm{~V}$, cuando se expone a una carga, ésta baja su tensión hasta $12,7 \mathrm{~V}$ y posteriormente la potencia disminuye debido al consumo constante. Con esa información se determina el tiempo de autonomía de la batería de 18 horas. Por lo tanto, a las 18 horas el sistema se desconecta y se evidencia que el acumulador disminuye su voltaje hasta $11,8 \mathrm{~V}$.
Figura 9. Perfil de carga de la batería 12V/100Ah.

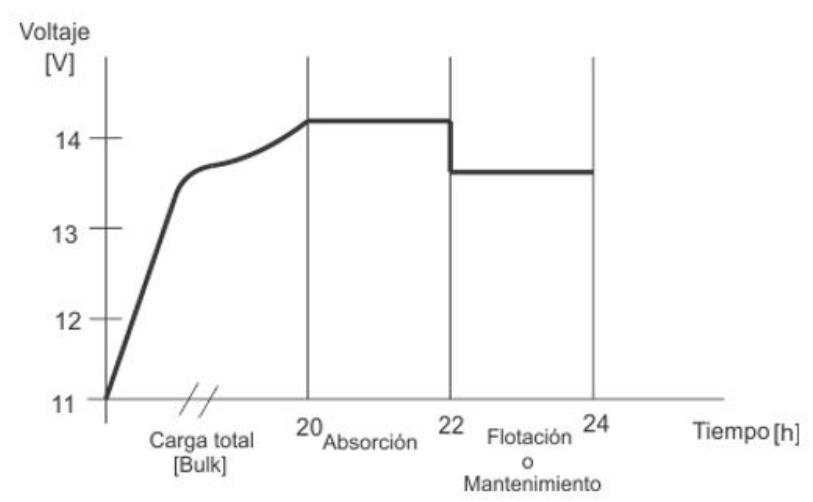

Figura 10. Perfil de descarga de la batería $12 \mathrm{~V} / 100 \mathrm{Ah}$.

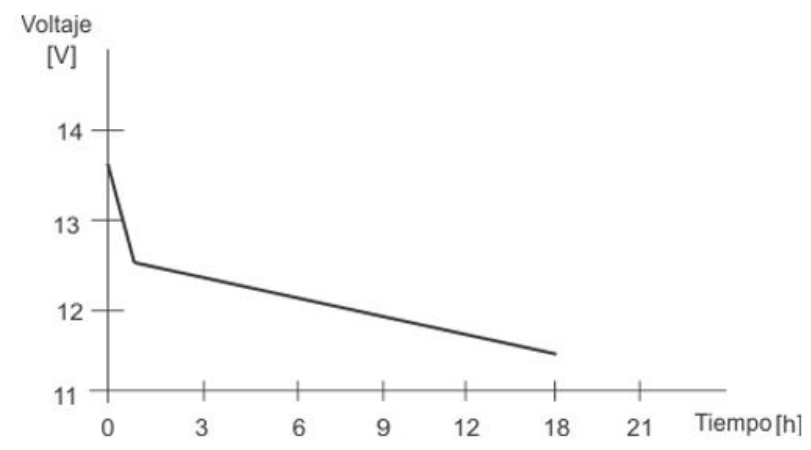

\section{Conclusión}

En este trabajo se construye e implementa un banco de pruebas para la turbina Francis donde se realizan ensayos en cinco aperturas de la válvula, obteniendo las curvas características de la bomba y se observa que cuando entrega menos de $400 \mathrm{~W}$ de potencia la turbina entrega 1000rpm. Estas 1000rpm no cargan la batería y por el contrario la carga resistiva conectada al controlador consume la energía de la batería, en cambio cuando se entrega una potencia superior a $400 \mathrm{~W}$ ésta entrega más de $1000 \mathrm{rpm}$ y la potencia generada eleva considerablemente su valor medido, por consiguiente, para generar se debe entregar más de $400 \mathrm{~W}$ de potencia disponible que en cabeza equivale a $5 \mathrm{~m}$.

La utilización de un controlador MPPT en un sistema de micro generación hidráulica protege y extiende la vida útil de las baterías, aunque este controlador se implementa para sistemas fotovoltaicos, el controlador funciona en sistemas de micro generación, para utilizarse se debe implementar un sistema que convierta la señal de $\mathrm{AC}$ a DC y después se rectifica ya que si no se implementa este sistema no funciona el controlador debido a que la tensión del generador es pulsante y variante.

\section{References}

[1] US Department of Energy, "Microhydropower Systems," 2017. 
[2] Y. E. A. Eldahab, N. H. Saad, and A. Zekry, "Enhancing the design of battery charging controllers for photovoltaic systems," Renewable and Sustainable Energy Reviews, vol. 58, pp. 646 - 655, 2016.

[3] D. Scott, "Microhydro: clean power from water," New Society Publishers, Canada, pp. 57-66, 2003.

[4] W. Shengjun, X. Qingshan, L. Qun, Y. Xiaodong, and C. Bing, "Optimal ev charging control strategy based on dc microgrid," Energy Procedia, vol. 100, pp. 243-247, 2016.

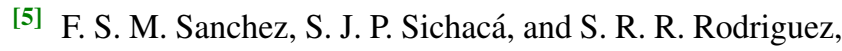
"Formulación de funciones de costo de incertidumbre en pequeñas centrales hidroeléctricas dentro de una microgrid," Ingenierías USBmed, vol. 8, no. 1, pp. 29-36, 2017.

[6] J. B. Franzini, E. J. Finnemore, and B. Lambert, Mecánica de Fluidos con aplicaciones en Ingeniería. McGraw-Hill, 1999.

[7] G. Jaramillo, "Laboratorio Pequeñas Centrales Hidroeléctricas."

${ }^{[8]}$ E. Hernández, "Máquinas hidráulicas, bombas centrifugas." Universidad Pontificia Bolivariana, 2013.

[9] E. Hernández, "Máquinas hidráulicas, turbinas hidráulicas.” Universidad Pontificia Bolivariana, 2013.

${ }^{[10]}$ Endesa, "Educa, central hidroeléctrica." 